\title{
Severe diabetic retinopathy at presentation in a young man
}

\author{
G.C. Morris, R.A. James, R.G. Powell and M.K. Jones \\ Department of Medicine, Ward 11, Singleton Hospital, Swansea SA2 8QA, UK
}

Summary: A 22 year old man presented with a severe ischaemic diabetic retinopathy and, in spite of
photocoagulation therapy, was blind in one eye 16 months after diagnosis. Four similar cases of aggressive
diabetic retinopathy are reviewed. There is increasing evidence that a small group of young men with
insulin dependent diabetes (IDD) may develop severe diabetic retinopathy at, or soon after, diagnosis.

\section{Introduction}

Many factors have been suggested as associated with the development of diabetic retinopathy. Duration of diabetes ${ }^{1}$ is the best recognized, while mean clinic blood glucose concentrations are high, particularly in those with severe retinopathy.,2 Other factors implicated in the development of diabetic retinopathy include male sex, ${ }^{3}$ cigarette smoking, ${ }^{4,5}$ hypertension, ${ }^{3}$ hyperlipidaemia ${ }^{6}$ and alcohol consumption. ${ }^{7}$ Patients with non-insulin dependent diabetes (NIDD) may have severe diabetic retinopathy at presentation of their disease $^{8}$ possibly associated with prolonged, asymptomatic hyperglycaemia. Severe retinopathy is exceptionally rare, however, in young patients with insulin dependent diabetes (IDD). ${ }^{9}$

We report here a young man who presented as an emergency with a severe pre-proliferative retinopathy and was found to be in mild diabetic ketosis. Diabetic eye disease progressed rapidly and within 9 months of presentation he was registered as partially sighted. Similar cases of aggressive diabetic retinopathy in young men with IDD are reviewed.

\section{Case report}

A 22 year old unemployed storeman was admitted as an emergency with a 10-day history of a painful, red right eye, headache and photophobia. On direct questioning he gave a history of thirst, polydipsia and polyuria. He had smoked 30 cigarettes/day for many years but had a low alcohol intake. There was no family history of diabetes mellitus.

On examination he was thin, ketotic, with a blood pressure of $130 / 70 \mathrm{mmHg}$. An acute ischae-

Correspondence: G.C. Morris, M.B., M.R.C.P.

Accepted: 22 August 1990 mic iritis was diagnosed and fundoscopy revealed an ischaemic diabetic retinopathy in both eyes. There were no other abnormal physical signs; in particular, no other complications of diabetes. Investigations revealed diabetic ketoacidosis with a random plasma glucose of $26.2 \mathrm{mmol} / 1$ and an arterial $\mathrm{pH}$ of 7.27. Fasting lipid levels were later found to be normal. He was initially treated with an infusion of intravenous insulin and normal saline and was subsequently transferred to twice daily subcutaneous Insulutard and Velosulin insulins. The iritis responded quickly to dexamethasone (Maxidex) and atropine eye drops.

Two months after diagnosis our patient had developed widespread flat retinal new vessels in all four quadrants of his right retina. There were cotton wool spots at both macular areas and ischaemic maculopathy accounted for reduced visual acuities of 6/36 in the right eye (previously $6 / 9$ at presentation) and $6 / 18$ in the left eye (previously 6/9). The right eye was treated with pan-retinal Argon laser photocoagulation. One month later new retinal vessels were noted in the left eye, and the right eye had developed optic disc new vessels (Figure 1). Both eyes received further laser therapy involving pan-retinal photocoagulation. The following month he developed rubeosis and thrombotic glaucoma presenting with pain and loss of vision in the right eye. Medical therapy lowered the intra-ocular pressure and further bilateral photocoagulation was applied.

The right thrombotic glaucoma was further controlled by peripheral retinal cryotherapy and cyclo-cryotherapy, but one week later there was progressive right vitreal haemorrhage. At this stage, 7 months after presentation, iris and optic disc new vessels were noted in the left eye and these were then treated with peripheral retinal cryotherapy. Our patient's vision had deteriorated to such an extent that he could detect only hand movements in the right eye due to vitreous haemor- 


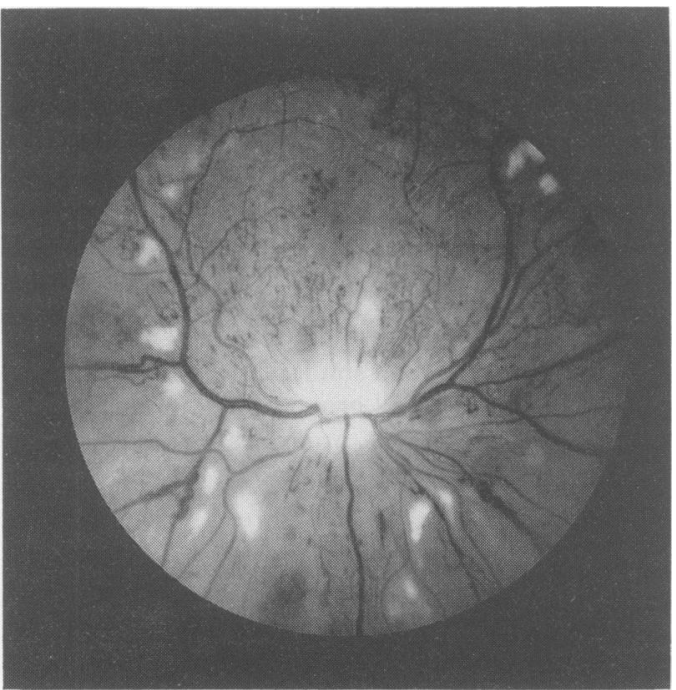

Figure 1 Ischaemic retinopathy with cotton wool spots, early new vessels and macular oedema in the left eye 3 months after presentation.

rhage and glaucoma, and vision in the left eye was only $6 / 36$ due to ischaemic maculopathy. He was registered as partially sighted. Two months later the left eye developed a vitreous haemorrhage which resolved spontaneously. No further treatment was applied. When last reviewed, 16 months after presentation, the right eye was completely blind whilst in the left eye vision had improved to $6 / 12$ with regression of optic disc new vessels and no evidence of glaucoma.

\section{Discussion}

The presenting feature of acute iritis in our patient was presumably related to the severity of the ocular ischaemia. Ischaemia has been previously identified as a cause of iritis in aortic arch syndrome and internal carotid artery stenosis. ${ }^{10}$ This ischaemic iritis is often followed by rubeosis, as in our case. Sixteen months after presentation our patient had total destruction of one eye, while extensive therapy had stabilized the other eye, which appeared to be maintaining useful vision. Our patient is unusual because of the presence of severe diabetic retinopathy at presentation of his disease.

Diabetic retinopathy is rarely seen within 5 years of diagnosis of IDD in young patients ${ }^{11}$ as the development of retinopathy is related to duration of disease. ${ }^{1,9}$ In children under 15 years of age who had been diabetic for less than 5 years, even minor diabetic retinopathy changes identified by fluoro- scein angiography were rarely observed..$^{9}$ In a series $\stackrel{\varrho}{\varrho}$ of over 5,000 newly diagnosed diabetics only $1.5 \%$ of patients in the 20-39 year age group had $\varrho$ retinopathy, and of these 9 young. patients with $\stackrel{\complement}{\complement}$ retinopathy, 7 were controlled on diet or oral $\overrightarrow{\vec{F}}$ hypoglycaemic therapy and only 2 had IDD. None $\frac{?}{0}$ of the patients had evidence of an accelerated type $\frac{\bar{C}}{O}$ of retinopathy, but of these 9 young patients, $8 \frac{\bar{\sigma}}{\bar{c}}$. were male.

Severe diabetic retinopathy at presentation of $\cong$ IDD in young patients has been described on two $\approx$ previous occasions ${ }^{12,13}$ while our patient is the fifth $\overrightarrow{0}$ reported case of severe proliferative retinopathy $\overrightarrow{ }$ presenting at, or shortly after, diagnosis in young $\overrightarrow{\mathcal{O}}$ patients. ${ }^{8,12,13}$ At least two, including our patient, $\frac{}{0}$ were heavy cigarette smokers, ${ }^{13}$ one was hyperten- $\frac{0}{3}$ sive, one gave a history of excessive alcohol intake, ${ }^{13}$ and one had been involved in severe. trauma prior to presentation of diabetes. ${ }^{12}$ All $5 \stackrel{\circ}{\circ}$ patients have been young men and men are recognized as being at increased risk of developing $\vec{c}$ proliferative diabetic retinopathy. ${ }^{3}$ Of the other 0 factors implicated in the development of severe diabetic retinopathy, ${ }^{3-7}$ only heavy cigarette smok- $\vec{T}$ ing was implicated in our case.

In two of the three cases of severe diabetic retinopathy in which details of follow-up are given, the retinopathy advanced rapidly in spite of phot $\Theta \vec{\theta}$ coagulation therapy, as in our case. In the four $\&$ case, yttrium implantation resulted in an improve: ment in the retinopathy, although photocoagula tion was subsequently necessary. Diabetic eye disease may progress rapidly in such patients, and $\overline{0}$ aggressive therapy should be considered at an early $\stackrel{2}{\circ}$

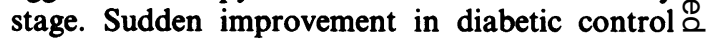
should, however, perhaps be avoided as dramatic $\overrightarrow{\overrightarrow{0}}$ improvement in glycaemic control might be assoc- 3 iated with a deterioration in retinopathy. ${ }^{14}$

Keen ${ }^{14}$ reviewed three trials in which continuous? subcutaneous insulin infusion was used to controlo diabetes intensively in patients with retinopathy. Results pointed towards accelerated worsening of $\frac{}{3}$ retinopathy in those intensively treated compared. to those on normal insulin regimes. Our patient's 0 insulin dependent diabetes was well controlled quickly as were Cove's ${ }^{13}$ second and Alexander's ${ }^{12}$ 을 patients and in each the retinopathy progressed $\supset$ rapidly within the first few months leading to blindness in two. This is in contrast to thosen reviewed by Keen where "little if any serious" deterioration of vision accompanied normogly- $N$ caemic re-entry'.

There is increasing evidence that a small group of young men with diabetes are prone to developo severe diabetic retinopathy. Review of our case and $\bar{\varnothing}$ similar cases suggests that accelerating factors in $\stackrel{?}{+}$ this group may be smoking, alcohol abuse, hyper- $\frac{T}{T}$ tension or previous severe trauma. 


\section{References}

1. Pirart, J. Diabetes mellitus and its degenerative complications: a prospective study of 4,400 patients observed between 1947 and 1973. Diabetes Care 1978, 1: 168-188, 252-263.

2. Groop, L.C., Teir, H., Koskimies, S. et al. Risk factors and markers associated with proliferative retinopathy in patients with insulin dependent diabetes. Diabetes 1986, 35: 1397-1403.

3. Bodansky, H.J., Cudworth, A.G., Drury, P.L. \& Kohner, E.M. Risk factors associated with severe proliferative retinopathy in insulin dependent diabetes mellitus. Diabetes Care 1982, 5: 97-100.

4. Muhlhauser, I., Sawicki, P. \& Berger, M. Cigarette smoking as a risk factor for macroproteinuria and proliferative retinopathy in Type I (Insulin dependent) diabetes. Diabetologia 1986, 29: 500-502.

5. Paetkau, M.E., Boyd, T.A.S., Winship, B. \& Grace, M. Cigarette smoking and diabetic retinopathy. Diabetes 1977 , 26: 46-49.

6. Weber, B., Burger, W., Hartmann, R., Hovener, G., Malchus, R. \& Oberdisse, U. Risk factors for the development of retinopathy in children and adolescents with Type I (Insulin dependent) diabetes mellitus. Diabetologia 1986, 29: 23-29.

7. Young, R.J., McCulloch, D.K., Prescott, R.J. \& Clarke, B.F. Alcohol - another risk factor for diabetic retinopathy? $\mathrm{Br}$ Med J 1984, 288: 1035-1037.
8. Soler, N.G., Fitzgerald, M.G., Malins, J.M. \& Summers, R.O.C. Retinopathy at diagnosis of diabetes with special reference to patients under 40 years of age. $\mathrm{Br} M e d J$ 1969, 3: 567-569.

9. Burger, W., Hovener, G., Dusterhus, R., Hartmann, R. \& Weber, B. Prevalence and development of retinopathy in children and adolescents with Type I (Insulin dependent) diabetes mellitus. A longitudinal study. Diabetologia 1986, 29: $17-22$.

10. Knox, D.L. Ischaemic ocular inflammation. Am J Ophthalmol 1976, 60: 995-1002.

11. Colwell, J.A. Effect of diabetic control on retinopathy. Diabetes 1966, 15: 497-499.

12. Alexander, W.D., Kearns, M., Kohner, E.M. \& Asplin, C.M. Trauma and severe proliferative retinopathy in diabetes mellitus. Br Med J 1979, 279: 831.

13. Cove, D.H. \& Hill, R.D. Severe retinopathy at presentation of insulin dependent diabetes mellitus. Practical Diabetes 1986, 3: 218

14. Keen, H. Normoglycaemic re-entry and diabetic complications. Diabetic Med 1984, 1: 85-87. 